Mathematical Research Letters 2, 791-795 (1995)

\title{
CLASSICAL VERSUS FREE DOMAINS OF ATTRACTION
}

\author{
H. Bercovici And V. Pata
}

\begin{abstract}
The domains of attraction of stable laws in Voiculescu's free probability theory are determined, and they are compared with the corresponding domains of attraction in classical probability theory. It is shown that each free domain of attraction is equal to some classical domain of attraction, but the corresponding attracting laws are quite different. Conversely, every classical domain of attraction occurs as a free domain of attraction.
\end{abstract}

Denote by $\mathcal{M}$ the family of all probability measures defined on the real line $\mathbf{R}$. Two measures $\mu, \nu$ in $\mathcal{M}$ will be said to be equivalent if there exist real numbers $a, b$, with $a>0$, such that $\mu(\sigma)=\nu(a \sigma+b)$ for every Borel set $\sigma \subset \mathbf{R}$. We will write $\mu \sim \nu$ if $\mu$ and $\nu$ are equivalent, and we denote by $[\nu]$ the equivalence class of $\nu$. On the set $\mathcal{M}$ there are defined two associative composition laws denoted $*$ and $\boxplus$. The measure $\mu * \nu$ is the classical convolution of $\mu$ and $\nu$. In probabilistic terms, $\mu * \nu$ is the probability distribution of $X+Y$, where $X$ and $Y$ are (commuting) independent random variables with distributions $\mu$ and $\nu$, respectively. The measure $\mu \boxplus \nu$ is the free (additive) convolution of $\mu$ and $\nu$ introduced by Voiculescu [10] (for compactly supported measures; free convolution was extended by Maassen [7] to measures with finite variance and by Bercovici and Voiculescu [2] to the whole class $\mathcal{M}$ ). Thus, $\mu \boxplus \nu$ is the probability distribution of $X+Y$, where $X$ and $Y$ are free random variables with distributions $\mu$ and $\nu$, respectively.

An important class of measures occurs in connection with the study of the limit laws of probability. A measure $\mu$ which is not a point mass will be said to be $*$-stable (resp. $\boxplus$-stable) if for every $\nu_{1}, \nu_{2} \in \mathcal{M}$ such that $\nu_{1} \sim \mu \sim \nu_{2}$ it follows that $\nu_{1} * \nu_{2} \sim \mu$ (resp. $\nu_{1} \boxplus \nu_{2} \sim \mu$ ). The stable measures were determined by Lévy (cf. [5]) in the classical context and by Bercovici and Voiculescu [2] in the context of free convolution.

Received September 29, 1995.

The first author was partially supported by a grant from the National Science Foundation. 
Before stating the main property of stable distributions we need one more definition. If $\mu, \nu \in \mathcal{M}$, we will say that $\mu$ belongs to the $*$-domain (resp. $\boxplus$-domain) of attraction of $\nu$ if there exist measures $\mu_{1}, \mu_{2}, \ldots$ such that

(i) $\mu_{n} \sim \underbrace{\mu * \mu * \cdots * \mu}_{n \text { times }}$ (resp. $\mu_{n} \sim \underbrace{\mu \boxplus \mu \boxplus \cdots \boxplus \mu}_{n \text { times }}$ ), and

(ii) $\mu_{n}$ converges weakly to $\nu$, i.e., $\int_{-\infty}^{\infty} f(t) d \mu_{n}(t) \rightarrow \int_{-\infty}^{\infty} f(t) d \mu(t)$ for every bounded continuous function $f$.

We will indicate this relationship between $\mu$ and $\nu$ by writing $\mu \in \mathcal{D}_{*}(\nu)$ (resp. $\mu \in \mathcal{D}_{\boxplus}(\nu)$ ). Clearly $\mathcal{D}_{*}(\nu)$ and $\mathcal{D}_{\boxplus}(\nu)$ only depend on the equivalence class of $\nu$, and hence we can define the sets $\mathcal{D}_{*}([\nu])=\mathcal{D}_{*}(\nu)$ and $\mathcal{D}_{\boxplus}([\nu])=\mathcal{D}_{\boxplus}(\nu)$. Observe that a stable law belongs to its own domain of attraction, and in fact the following is true.

1. Theorem. Assume that $\mu \in \mathcal{M}$ is not a point mass. Then $\mu$ is $*$-stable (resp., $\boxplus$-stable) if and only if $\mathcal{D}_{*}(\nu)$ (resp., $\mathcal{D}_{\boxplus}(\nu)$ ) is not empty.

(The definition of stable laws, and the above result, could be extended to include point masses, but there are good reasons for not doing so. Convergence to a point mass is the object of laws of large numbers.)

Theorem 1 was proved by Lévy (cf. [5]) in the classical case and by Pata [8] in the free case.

The main result we want to announce here is as follows.

2. Theorem. A measure $\mu \in \mathcal{M}$ belongs to $a *$-domain of attraction if and only if it belongs to $a \boxplus$-domain of attraction. More precisely, there exists a bijection $[\nu] \leftrightarrow\left[\nu^{\prime}\right]$ between equivalence classes of $*$-stable laws $[\nu]$ and equivalence classes of $\boxplus$-stable laws $\left[\nu^{\prime}\right]$ such that $\mathcal{D}_{*}([\nu])=\mathcal{D}_{\boxplus}\left(\left[\nu^{\prime}\right]\right)$.

To illustrate the theorem, let us consider the case when $\nu$ is Gaussian. The corresponding $\boxplus$-stable law $\nu^{\prime}$ is the semicircle law (i.e., an absolutely continuous measure on $[-1,1]$ with density $\left.2 \sqrt{1-t^{2}} / \pi, t \in[-1,1]\right)$ as shown by Voiculescu's free central limit theorem [10]. The free central limit theorem was extended to measures with finite variance by Maassen [7]. Finally, Pata [9] proved that in fact $\mathcal{D}_{*}([\nu])=\mathcal{D}_{\boxplus}\left(\left[\nu^{\prime}\right]\right)$ in this case.

An analogous result for the weak law of large numbers was established in [6] and [1]. Namely, it was shown that the classical weak law of large numbers holds for a measure $\mu$ if and only if the free weak law of large numbers holds for $\mu$. The necessary and sufficient condition for the classical weak law of large numbers was given by Kolmogorov in terms of tail-sums. It is as follows:

$$
\lim _{t \rightarrow \infty} t \mu(\{x:|x|>t\})=0 .
$$


The characterization of probability measures in a domain of attraction involves regularly varying functions in the sense of Karamata (see [3] for a comprehensive account). We recall the basic definitions. A function $f$ : $(0,+\infty) \rightarrow(0,+\infty)$ is said to be slowly varying if $\lim _{x \rightarrow \infty} f(t x) / f(x)=1$ for every $t>0$. A function $F:(0,+\infty) \rightarrow(0,+\infty)$ is said to be regularly varying with index $\beta \in \mathbf{R}$ if $f(x)=x^{-\beta} F(x)$ is a slowly varying function.

The following result is a characterization of domains of attraction (see for instance [4] for the classical case).

3. Theorem. A measure $\mu \in \mathcal{M}$ belongs to a classical (resp. free) domain of attraction if and only if the following two conditions are satisfied:

(1) The function $F(x)=\mu((-x, x))$ varies regularly with exponent $2-$ $\alpha$, where $\alpha$ is some number in $(0,2]$;

(2) If $\alpha \neq 2$ then the limit

$$
p=\lim _{x \rightarrow \infty} \frac{\mu((x,+\infty))}{\mu((-\infty,-x))+\mu((x,+\infty))}
$$

must exist.

If (1) and (2) are satisfied, then the class $[\nu]$ (resp. [ $\left.\nu^{\prime}\right]$ ) such that $\mu \in$ $\mathcal{D}_{*}([\nu])$ (resp. $\left.\mu \in \mathcal{D}_{\boxplus}\left(\left[\nu^{\prime}\right]\right)\right)$ is uniquely determined by the numbers $\alpha \in$ $(0,2]$ and $p \in[0,1]$.

As mentioned above, the classical version of Theorem 3 is well known. In our approach we first prove Theorem 3 in the free case, and then deduce Theorem 2 as an immediate corollary. It seems very desirable to obtain a more direct proof of Theorem 2. This might unveil a new link between free and classical convolutions.

The classical results on stable laws can be approached in several ways, the most powerful of which involves Fourier analysis. An analogue of the Fourier transform for free convolution was discovered by Voiculescu [11], originally for measures with compact support. This was extended in [7] to measures with finite variance, and in [2] to the whole class $\mathcal{M}$. Our proof of Theorem 3 involves this analogue of the Fourier transform and therefore we provide a brief description of the technique. Given $\mu \in \mathcal{M}$ one considers analytic functions $G_{\mu}, F_{\mu}:\{z \in \mathbf{C}: \Im z>0\} \rightarrow \mathbf{C}$ defined by

$$
G_{\mu}(z)=\int_{-\infty}^{\infty} \frac{1}{z-t} d \mu(t)
$$

and $F_{\mu}(z)=1 / G_{\mu}(z)$. The function $F_{\mu}(z)$ is close to $z$ if $|z| \rightarrow \infty$ in such a way that $\Re z / \Im z$ stays bounded. Thus $F_{\mu}$ has an inverse $F_{\mu}^{-1}$ defined, 
say, in $\Omega_{M}=\{z: \Im z>M,|\Re z|<\Im z\}$ for sufficiently large $M$. We can then define the function $\phi_{\mu}(z)=F_{\mu}^{-1}(z)-z$ which is analytic and has nonpositive imaginary part in $\Omega_{M}$. The function $\phi_{\mu}$ - which we will call the Voiculescu transform of $\mu$ - linearizes free convolution in the sense that, for $\mu, \nu \in \mathcal{M}, \phi_{\mu \boxplus \nu}=\phi_{\mu}+\phi_{\nu}$ in the common domain of the three functions involved.

The $\boxplus$-stable measures $\mu \in \mathcal{M}$ can be classified using a parameter $\alpha \in$ $(0,2]$, and their Voiculescu transforms are as follows.

(i) If $\alpha \in(0,1), \phi_{\mu}(z)=a+b z^{-\alpha+1}$ with $a \in \mathbf{R}, b \neq 0$, and $\arg b \in$ $[\pi,(1+\alpha) \pi]$.

(ii) If $\alpha=1, \phi_{\mu}(z)=a+b \log z$ or $\phi_{\mu}(z)=a+b(i \pi-\log z)$, where $\Im a \leq 0$ and $b \in(-\infty, 0]$.

(iii) If $\alpha \in(1,2], \phi_{\mu}(z)=a+b z^{-\alpha+1}$ with $a \in \mathbf{R}, b \neq 0$, and $\arg b \in$ $[(\alpha-2) \pi, 0]$.

Observe that for $\alpha=2, b$ must be real and we get only one class of stable distributions, namely the class of all semicircle laws. If $\alpha=1$ and $b=0$ one obtains the Cauchy laws which are also stable of index one in the classical case. We do not know whether there are any other measures which are both $*$-stable and $\boxplus$-stable.

The preceding description of the $\boxplus$-stable laws was given in Theorem 7.5 of [2]. (the statement of that theorem is somewhat incomplete since it does not include the measures with $\phi_{\mu}(z)=a+b(i \pi-\log z)$; these are reflections in the origin of the measures with Voiculescu transforms $a+b \log z$.)

We will give now a brief sketch for the proof of Theorem 3. The more difficult direction is the proof that a probability measure which belongs to a $\boxplus$-domain of atraction satisfies conditions (1) and (2) in the statement. Fix therefore $\mu, \nu \in \mathcal{M}$ such that $\nu$ is $\boxplus$-stable, and assume that $\mu \in \mathcal{D}_{\boxplus}(\nu)$. The scaling properties of the Voiculescu transform imply the existence of cconstants $A_{n}, B_{n} \in \mathbf{R}, B_{n}>0$, such that

$$
\frac{n}{B_{n}} \phi_{\mu}\left(B_{n} z\right)+A_{n} \rightarrow \phi_{\nu}(z) \text { as } n \rightarrow \infty
$$

uniformly on the compact subsets of $\Omega_{M}$. This convergence relation can be converted into a statement about the asymptotic behavior of the function $G_{\mu}$ in $\Omega_{M}$. The real and imaginary parts of $G_{\mu}$, i.e. the integrals

$$
\int_{-\infty}^{\infty} \frac{x-t}{(x-t)^{2}+y^{2}} d \mu(t), \int_{-\infty}^{\infty} \frac{-y}{(x-t)^{2}+y^{2}} d \mu(t)
$$

can then be analyzed using the Tauberian theory of Karamata in order to yield the desired conclusion about the measure $\mu$. The technical details of 
the proof are rather delicate, and will be described in a separate paper. We will only point out here that the behavior of the imaginary part of $G_{\mu}$ is closely related with the regular variation condition (1) of Theorem 3 , while the behavior of the real part yields the tail-balancing condition (2).

\section{References}

1. H. Bercovici and V. Pata, The law of large numbers for free identically distributed random variables, Ann. of Probability (to appear).

2. H. Bercovici and D. Voiculescu, Free convolution of measures with unbounded support, Indiana U. Math. J. 42 (1993), 733-773.

3. N.H. Bingham, C.M. Goldie, and J.L. Teugels, Regular variation, Cambridge Univ. Press, Cambridge, 1987.

4. W. Feller, An introduction to probability theory and its applications, Vol. II, John Wiley \& Sons, New York, 1971.

5. P. Lévy, Théorie de l'addition des variables aléatoires, Gauthier-Villars, Paris, 1937.

6. J.M. Lindsay and V. Pata, Some weak laws of large numbers in non-commutative probability, Math. Z. (to appear).

7. H. Maassen, Addition of freely independent random variables, J. Funct. Anal. 106 (1992), 409-438.

8. V. Pata, Lévy type characterization of stable laws for free random variables, Trans. Amer. Math. Soc. 347 (1995), 2459-2474.

9. $\quad$ The central limit theorem for free additive convolution, J. Funct. Anal. (to appear).

10. D. Voiculescu, Symmetries of some reduced free product $C^{*}$-algebras, Operator algebras and their connections with topology and ergodic theory, Lecture Notes in Mathematics, No. 1132, Springer Verlag, New York, 1985, pp. 556-588.

11. Addition of certain non-commuting random variables, J. Funct. Anal. 66 (1986), 323-346.

Mathematics Department, Indiana University, Bloomington, IN 47405

E-mail address: bercovic@indiana.edu ITALY

Dipartimento di Matematica "F. Enriques", Università di Milano, Milano,

E-mail address: vpata@vmimat.mat.unimi.it 